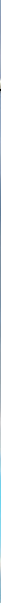

\title{
Veränderung der Versorgungsabläufe ist nicht qualitätsgetrieben
}

Wir leben in einer ereignisreichen Zeit, in der uns die Entwicklung der pharmazeutischen Forschung in kurzen Abständen mit Zulassungen neuer Medikamente bereichert - darunter Zytostatika, Tyrosinkinaseinhibitoren oder Immuntherapeutika. Für die Patienten können sich mit der Verfügbarkeit neuer Substanzen deutliche Verbesserungen im Leben mit der Krebserkrankung ergeben. Einige Wirkstoffe können den Betroffenen bis dato nicht erreichte Überlebenszeiten ermöglichen. Bei anderen Präparaten ist wiederum nur mit einem geringen Zusatznutzen für die Patienten zu rechnen.

\section{Maßnahmen gegen „Mondpreise"?}

Mit den neuen Zulassungen kommen überwiegend teure Substanzen auf die Solidargemeinschaft zu, deren Preise von den Herstellern im ersten Jahr selbst festgelegt werden. Diese von den Krankenkassen als „Mondpreise“ benannten Kosten haben in Deutschland auf Druck der Krankenkassen zu einer Initiative des Bundesgesundheitsministers Hermann Gröhe geführt. In dem sogenannten Pharmadialog mit der pharmazeutischen Industrie wurden Kostenbremsen diskutiert, die im Sommer in einer Gesetzesinitiative münden sollen. So soll etwa rückwirkend ein Erstattungsbeitrag erhoben werden, wenn der Umsatz eines Medikamentes im ersten Jahr mehrere hundert Millionen Euro überschreitet.

Wie sich diese und andere Mechanismen auswirken werden, bleibt abzuwarten. Die Krankenkassen reagieren bereits im Angesicht der massiv steigenden Kosten und fürchten, Kostensteigerungen in Form von Zusatzbeiträgen an ihre Versicherten weitergeben zu müssen.

\section{Kostenersparnis zu Lasten der Behandler}

Zusätzlich sollen Sparpotenziale an verschiedenen Enden ausgeschöpft werden. So wurde die Hilfsmittelverordnung durch einige Kassen verändert und erfordert aufwändige Antragsverfahren, die die Versorgung der schwerstkranken Patienten mit Schmerz- und Ernährungspumpen aktuell erschwert.

Nach einem Testlauf in Berlin wird die Versorgung der vertragsärztlich onkologisch tätigen Kollegen mit Zytostatika auch in weiteren Bundesländern unter Kostengesichtspunkten aus-

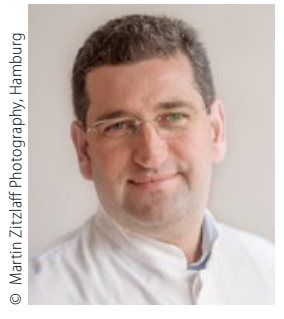

PD Dr. med. Gunter Schuch

Hämatologisch-Onkologische Praxis Altona, Hamburg

gunter.schuch@hopa-hamburg.de geschrieben. Das führt $\mathrm{zu}$ erheblichem organisatorischem Mehraufwand und zu veränderten Versorgungsabläufen zu Lasten der versorgenden Ärzte. Damit führt der Kostendruck auf Seiten der Krankenkassen durch einseitige Maßnahmen zu einer Veränderung der Versorgungsabläufe, die nicht qualitätsgetrieben ist.

\section{Herausforderungen nur gemeinsam zu meistern}

Man muss hoffen, dass die Herausforderungen, denen sich die Onkologie gegenüber sieht, nunmehr in einer gemeinsamen $\mathrm{Ab}$ stimmung aller relevanten gesellschaftlichen Bereiche im Sinne der Solidargemeinschaft bewältigt werden - das heißt durch Politik, Krankenkassen, Ärzteverbände, Industrie, Patienten und ethische Gruppierungen. Eine öffentlich geführte Diskussion sollte zum Fortbestehen unseres leistungsfähigen Gesundheitssystems beitragen, um eine implizite Rationierung innovativer Therapieverfahren zu vermeiden.

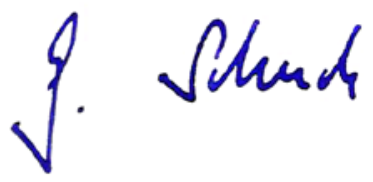

